\title{
Transient adrenal insufficiency and post-treatment bradycardia in scrub typhus - a case report
}

\author{
Sudhagar Mookkappan, Aneesh Basheer, Saranya Chidambaram, Nagarajan Natarajan, \\ Bhairappa Shrimanth
}

Department of General Medicine, Pondicherry Institute of Medical Sciences

\section{CASE REPORT}

Please cite this paper as: Mookkappan S, Basheer A, Chidambaram S, Natarajan N, Shrimanth B. Transient adrenal insufficiency and post-treatment bradycardia in scrub typhus - a case report. AMJ 2014, 7, 3, 164-167. http://doi.org/10.21767/AMJ.2014.1951

\section{Corresponding Author:}

Aneesh Basheer

Assistant Professor

Department of General Medicine, Pondicherry Institute of

Medical Sciences,

Ganapathichettikulam, Kalapet,

Pondicherry, India. 605014

Email: basheeraneesh@gmail.com

\section{ABSTRACT}

Scrub typhus appears throughout the Asia-Pacific rim. This disease is known for its diverse clinical manifestations and complications. There is no literature on the association of scrub typhus with adrenal insufficiency. Relative bradycardia has been reported in scrub typhus during the febrile phase but not during convalescence. We report the case of a 45 year-old woman with scrub typhus whose blood pressure was persistently low due to acute adrenal insufficiency. Adrenal failure responded to supplementation with steroids. She also developed sinus bradycardia during the afebrile period following treatment.

\section{Key Words}

Adrenal failure, scrub typhus, relative bradycardia, ACTH stimulation test

\section{What this report adds:}

\section{What is known about this subject?}

Scrub typhus is an infection with multisystem involvement. This infection and its complications respond very well to specific antimicrobial therapy with doxycycline or azithromycin.

\section{What is the key finding of this report?}

Transient adrenal failure may be a rare and treatable complication of scrub typhus.

\section{What are the implications for future practice?}

Patients with scrub typhus should be carefully monitored for hypotension or postural drop, and subjected to an ACTH stimulation test where needed, so that adrenal failure can be recognised early and treated. The significance of bradycardia during convalescence from scrub typhus needs further research.

\section{Background}

Scrub typhus is a mite-borne infectious disease caused by Orientia tsusugamushi, which was first described by the Chinese in the third century. The vector for scrub typhus is the larval trombiculid mite of the genus leptotrombidium. The disease occurs 7-10 days after the bite of an infected mite. ${ }^{1}$ Fever, headache, myalgia, rash, and eschar are common features. Relative bradycardia, which is an inappropriate low heart rate response to every degree rise in body temperature, is known to occur in scrub typhus. ${ }^{2}$ Our case represents a rare association of Addisonian crisis in scrub typhus and post-treatment bradycardia. Unless the clinician is aware of this rare possibility, hypotension in scrub typhus might easily be misdiagnosed as hypovolaemic or septic shock and delay institution of glucocorticoids that may be lifesaving.

\section{Case details}

A 45-year-old woman without co-morbidities presented with a history of high grade, intermittent fever over the previous five days, associated with chills and evening rise 
of temperature. She also complained of fatigue and giddiness while walking over the previous two days. There was no history of bleeding manifestations, rashes, chest pain, palpitation, abdominal pain, or altered bowel habits.

On examination she was conscious, oriented but dehydrated. She was pale and had oral candidiasis. An eschar was present in the pubic region (Figure 1). Her blood pressure was $80 / 60$ $\mathrm{mmHg}$ with postural drop of $20 \mathrm{mmHg}$; her heart rate was 100 beats/minute and respiratory rate 20/minute. Systemic examination was within normal limits.

\section{Figure 1: Eschar in the pubic region}

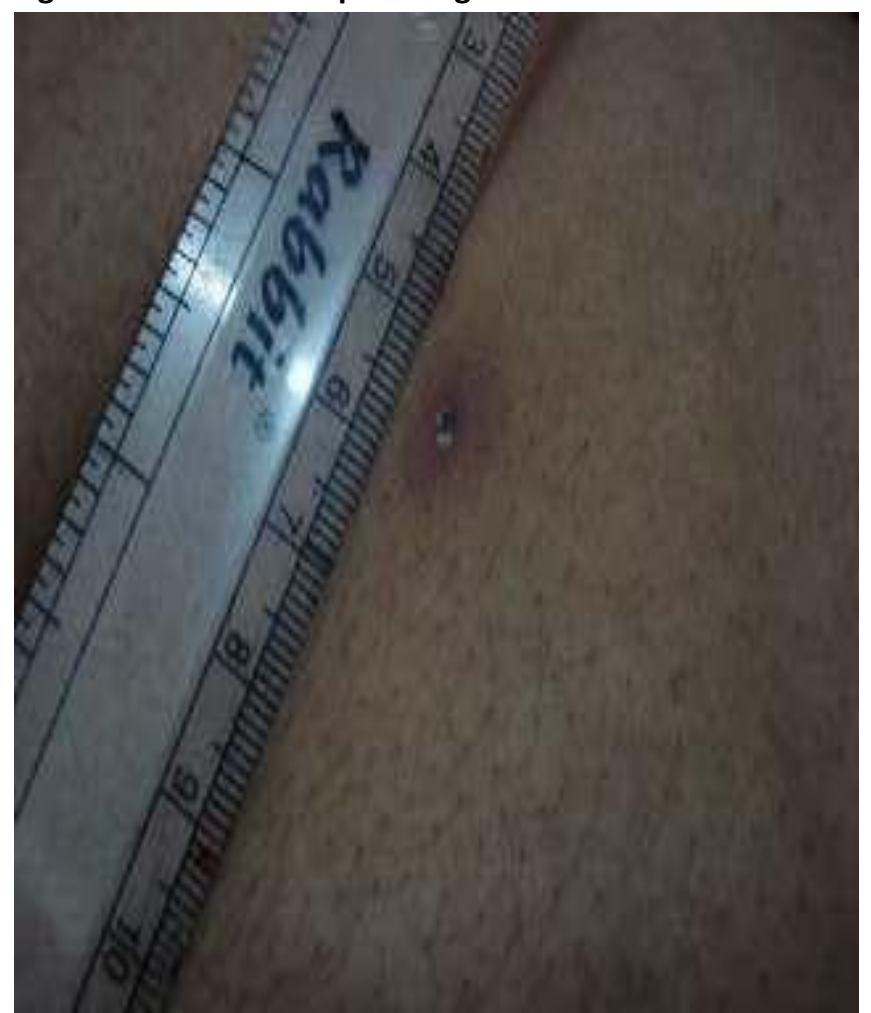

Initial investigations (Table 1) showed anaemia (8.5 gm/dl), thrombocytopenia (32,000/cu.mm), and leukocytosis (19800/cu.mm). Liver functions were impaired with an elevated serum bilirubin and transaminases. There was mild azotemia as well. Fasting blood glucose was $94 \mathrm{mg} / \mathrm{dl}$ with a glycated haemoglobin of 5.4 per cent (normal: < 5.5 per cent). Chest radiograph was normal and a 2D echocardiogram revealed mild pericardial effusion. Ultrasonogram of the abdomen showed normal sized kidneys with good corticomedullary differentiation.
Table 1: Laboratory investigations during the course of illness

\begin{tabular}{|c|c|c|c|c|}
\hline & Day 1 & Day 3 & Day 5 & Day 7 \\
\hline $\begin{array}{l}\text { Haemoglobin } \\
\text { (g/dl) }\end{array}$ & 8.5 & 8.5 & 9.2 & 9.4 \\
\hline $\begin{array}{l}\text { WBC count } \\
\text { (cells/cu.mm) }\end{array}$ & 19,800 & 15,200 & 8,600 & 5,200 \\
\hline $\begin{array}{l}\text { Differential } \\
\text { count }\end{array}$ & $\begin{array}{l}\mathrm{N}-76 \\
\mathrm{~L}-24\end{array}$ & $\begin{array}{l}\mathrm{N}-70 \\
\mathrm{~L}-30\end{array}$ & $\begin{array}{l}\mathrm{N}-45 \\
\mathrm{~L}-55\end{array}$ & $\begin{array}{l}N-58 \\
L-42\end{array}$ \\
\hline $\begin{array}{l}\text { Platelets } \\
\text { (cells/cu.mm) }\end{array}$ & 32,000 & 40,000 & $1,40,000$ & $2,60,000$ \\
\hline $\begin{array}{ll}\text { Blood urea } \\
(\mathrm{mg} / \mathrm{dl})\end{array}$ & 138 & 89 & 49 & 38 \\
\hline $\begin{array}{l}\text { S. Creatinine } \\
(\mathrm{mg} / \mathrm{dl})\end{array}$ & 1.7 & 1.4 & 0.9 & 0.9 \\
\hline AST (IU/L) & 190 & & 167 & 36 \\
\hline ALT (IU/L) & 132 & & 108 & 30 \\
\hline
\end{tabular}

The patient was hydrated with intravenous fluids. After obtaining blood and urine samples for culture, she was started empirically on ceftriaxone injection and oral azithromycin to cover for possible leptospirosis, scrub typhus, and enteric fever. IgM scrub ELISA was positive and hence, azithromycin was continued. Following this, the patient became afebrile, and the anaemia and thrombocytopenia showed improvement towards normal. However, her blood pressure continued to be $90 / 60$ $\mathrm{mmHg}$ with postural fall. An 8:00 am serum cortisol estimate was low, suggestive of adrenal insufficiency (2.9 $\mu \mathrm{g} / \mathrm{dL}$ ). Subsequently, an ACTH stimulation test was done, which did not show significant rise in serum cortisol after 30 and 60 minutes of ACTH administration, confirming the presence of adrenal insufficiency. She was started on intravenous steroids, after which her blood pressure improved. Later, she was put on oral steroids.

On the seventh day, the patient developed sinus bradycardia with a heart rate of $40-45$ beats per minute. Echocardiogram was normal. The patient was symptomatically treated with orciprenaline following which her heart rate returned to normal after two days. She was discharged on oral steroids. At her next visit, we started tapering steroids and by the end of the third week we were able to withdraw steroids completely without recurrence of postural hypotension.

In this case, the patient had a rare association of scrub typhus and adrenal insufficiency. Though the fever and haematological abnormalities improved with treatment of scrub typhus, adrenal insufficiency persisted in the form of hypotension. Bradycardia also developed after 
treatment when the patient was afebrile.

\section{Discussion}

Scrub typhus may begin insidiously with headache, anorexia, and malaise, or start abruptly with chills and fever. As the disease progresses, the fever may become high grade and severe prostration may develop. Some patients develop rash and eschar at the site of the chigger bite. The severity of infection may range from mild signs and symptoms to multiorgan failure. ${ }^{3}$

Relative bradycardia has been reported during the febrile phase in scrub typhus. ${ }^{2}$ This phenomenon is not specific to scrub typhus and, in fact, has been described classically with enteric fever. ${ }^{4}$ Unusually, our patient had tachycardia during the febrile phase of the disease and developed bradycardia two days after the fever subsided. No structural cardiac abnormality was demonstrable and electrocardiogram showed sinus rhythm. Unlike relative bradycardia that occurs during fever, this phenomenon of transient sinus bradycardia during the afebrile period has not been reported in the literature.

Common laboratory abnormalities encountered in scrub typhus include thrombocytopenia, leucopenia, or leucocytosis, elevated liver enzymes, elevated bilirubin, and creatinine. ${ }^{6}$ Our patient had all these abnormalities. These abnormalities responded positively to the treatment of scrub typhus in our patient. The preferred drug for scrub typhus is doxycycline and most patients become afebrile within 24-48 hours of treatment. Since our patient had de-ranged liver function tests, we chose to administer azithromycin, which is as effective as doxycycline for scrub typhus. ${ }^{5}$ It is also useful against drug-resistant strains. ${ }^{6}$

Meningitis, encephalitis, ARDS, acute renal failure, acute hearing loss, and pericardial effusion have been reported in patients with scrub typhus. ${ }^{7-9}$ Our patient had acute renal failure and mild pericardial effusion. These were expected complications that improved with azithromycin. However, our patient developed adrenal insufficiency that was unresponsive to treatment of scrub typhus. Since the patient remained symptom-free after withdrawal of steroids, we presume that she had transient adrenal insufficiency secondary to scrub typhus. Although it is possible that the patient could have had a pre-existing subclinical adrenal insufficiency decompensated by scrub typhus, the reversibility with short-term steroids and lack of any symptoms prior to the infection weighs heavily in favour of a transient phenomenon caused by scrub typhus. Further, objective evidence for this was the normal 8:00 am serum cortisol done four weeks after discontinuation of steroids.

To our knowledge adrenal insufficiency has not been reported in scrub typhus to date. Whether this is due to its rarity or under-recognition and under-reporting needs further exploration. Since the mechanism for most of the clinical manifestations and complications in scrub typhus is increased vascular permeability and perivasculitis of small blood vessels, ${ }^{10}$ we propose that transient adrenal failure may also be triggered by similar events in the adrenals. Though the other manifestations responded to antibiotic therapy, adrenal failure required steroid supplementation, following which the blood pressure returned to normal levels. Hence, it is reasonable to consider that the pathogenesis of transient adrenal failure observed in our patient was not vasculitis alone.

This case highlights two unreported and important issues regarding scrub typhus. First, transient adrenal insufficiency may complicate scrub typhus, though it has not been reported so far. Awareness of this complication might result in more meticulous observation of patients and more case reports in the future. Identification of this potentially fatal manifestation is important because, unlike most other complications of scrub typhus, it is unlikely to respond to antibiotic therapy alone and requires prompt administration of steroids. Moreover, in the setting of an infection, hypotension is likely to be treated with fluids and inotropes, rather than steroids, unless this rare possibility is also kept in mind. Second, patients with scrub typhus may develop bradycardia during their convalescence. Though our patient had sinus bradycardia, this aspect needs further study. We treated the patient with orciprenaline for two days. Whether treatment is really needed for such bradycardia episodes requires further investigation.

\section{References}

1. Sonthayanon P, Chierakul W, Wuthiekanun V, Phimda K, Pukrittayakamee S, Day NP, et al. Association of high Orientiatsutsugamushi DNA loads with disease of greater severity in adults with scrub typhus. J Clin Microbiol. 2009 Feb;47(2):430-4.

2. Aronoff DM, Watt G. Prevalence of relative bradycardia in Orientia tsutsugamushi infection. Am J Trop Med Hyg. 2003 Apr;68(4):477-9.

3. Sonthayanon $P$, Chierakul W, Wuthiekanun V, Blacksell SD, Pimda K, Suputtamongkol Y, et al. Rapid 
diagnosis of scrub typhus in rural Thailand using polymerase chain reaction. Am J Trop Med Hyg. 2006 Dec;75(6):1099-102.

4. Ostergaard L, Huniche B, Andersen PL. Relative bradycardia in infectious diseases. J Infect. 1996 Nov;33(3):185-91.

5. Phimda K, Hoontrakul S, Suttinont C, Chareonwat S, Losuwanaluk K, Chueasuwanchai S, et al. Doxycycline versus azithromycin for treatment of leptospirosis and scrub typhus. Antimicrob Agents Chemother. 2007 Sep;51(9):3259-63.

6. David HW, Stephen JD, Thomas M. Diseases caused by rickettsiae, mycoplasmas and chlamydiae. Harrison's Principles of Internal Medicine. Volume 1. 18th edition, 2012. 1413

7. Lee HC, Ko WC, Lee HL, Chen HY. Clinical manifestations and complications of rickettsiosis in southern Taiwan. J Formos Med Assoc. 2002 Jun;101(6):385-92.

8. Premaratna R, Chandrasena TG, Dassayake AS, Loftis AD, Dasch GA, de Silva HJ. Acute hearing loss due to scrub typhus: a forgotten complication of a reemerging disease. Clin Infect Dis. 2006 Jan 1;42(1):e6-8.

9. Silpapojakul K, Ukkachoke C, Krisanapan S, Silpapojakul K. Rickettsial meningitis and encephalitis. Arch Intern Med. 1991 Sep;151(9):1753-7.

10. Paris DH, Jenjaroen K, Blacksell SD, Phetsouvanh $R$, Wuthiekanun V, Newton PN et al. Differential patterns of endothelial and leucocyte activation in 'typhus-like' illnesses in Laos and Thailand. Clin Exp Immunol. 2008 Jul;153(1):63-7

\section{PEER REVIEW}

Not commissioned. Externally peer reviewed.

\section{CONFLICTS OF INTEREST}

The authors declare that they have no competing interests.

\section{PATIENT CONSENT}

The authors, Mookkappan S, Basheer A, Chidambaram S, Natarajan N, and Shrimanth B declare that:

1. They have obtained written, informed consent for the publication of the details relating to the patient(s) in this report.

2. All possible steps have been taken to safeguard the identity of the patient(s).

3. This submission is compliant with the requirements of local research ethics committees. 